IJAMSR 3 (1) www.ijamsr.com CrossRef: https://doi.org/10.31426/ijamsr.2020.3.1.3013

\title{
SPECTRAL COORDINATE CHANNEL AND COSINE FINDER ADDITIONALLY ACCEPT GAUSSIAN CONVEYANCES FOR THE OBJECTIVITY AND FOUNDATION OF PIXELS
}

\author{
Sunil Singarapu', Dr. Avinash Gour ${ }^{2}$ \\ ${ }^{1}$ Research Scholar, Dept. of Electronics and Communication Engineering, Sri Satya Sai University of \\ Technology \& Medical Sciences, Sehore, Bhopal-Indore Road, Madhya Pradesh, India. \\ ${ }^{2}$ Research Guide, Dept. of Electronics and Communication Engineering, Sri Satya Sai University of \\ Technology \& Medical Sciences, Sehore, Bhopal Indore Road, Madhya Pradesh, India \\ Email: sunil.sun0505@gmail.com
}

\section{Keywords:}

Gaussian Process, Hyper Spectral Image, Kernel, Reflectance, Matrix.

\begin{abstract}
A B S T R A C T
Comprehensive assessment using the hyper spectral datasets reveals the effective analyzes of spectral specifics from hyper spectral images by this review paper. The hyper spectral data sets are determined over the distorted Gaussian Processes related to those hyper spectral data sets and the resulting image is built by linking the structures with the unique Procedures. The Gaussian Processes are included in sets using groups of hyper spectral training data sets. In this paper we are going to review how to analyze spectral details from hyper spectral images using well-known spectral analyses by Gaussian Processes and combining them with the hyper spectral images. Each data set is distorted to match the spectral quantization of the test input hyper spectral image. This review and implementation utilize process Kernels to analyze the comparative smoothness of hyper spectral reflectance and eliminates errors in resulting signals for better estimation of the hyper spectral reflectance result. This review concludes the anticipated Gaussian Processes and combined kernel based analyzing hyper spectral reflectance.
\end{abstract}

Citation : Sunil Singarapu, Dr. Avinash Gour (2020). Spectral Coordinate Channel And Cosine Finder Additionally Accept Gaussian Conveyances For The Objectivity And Foundation Of Pixels. International Journal of Advanced Multidisciplinary Scientific Research (IJAMSR) ISSN:2581-4281, 3(1), January 2020, pp 23 - 43 
IJAMSR 3 (1) www.ijamsr.com CrossRef: https://doi.org/10.31426/ijamsr.2020.3.1.3013

\section{Introduction}

Ongoing advances remote sensing and geographic information has driven the path for the improvement of hyperspectral sensors. Hyperspectral far off detecting, otherwise called imaging spectroscopy, is a moderately new innovation that is as of now being examined by analysts and researchers as to the recognition and recognizable proof of minerals, earthly vegetation, and man-made materials and foundations [1][2]. Imaging spectroscopy has been utilized in the research center by physicists and scientists for more than 100 years for ID of materials and their structure. Spectroscopy can be utilized to identify singular ingestion includes because of explicit concoction bonds in a strong, fluid, or gas. As of late, with propelling innovation, imaging spectroscopy has started to concentrate on the Earth. The idea of hyperspectral far off detecting started in the mid-80 and to this point has been utilized most generally by geologists for the planning of minerals. Real recognition of materials is subject to the spectral inclusion, spectral goal, and sign to-commotion of the spectrometer, the wealth of the material and the quality of ingestion highlights for that material in the frequency locale estimated 3$][4]$.
Hyper spectral analyses consolidate imaging and spectroscopy in a solitary framework which frequently incorporates enormous informational indexes and require new processing techniques. Hyper spectral informational collections are commonly made out of around 100 to 200 spectral groups of generally slender transmission capacities (5-10 $\mathrm{nm}$ ), while, multispectral informational collections are normally made out of around 5 to 10 groups of moderately enormous data transfer capacities (70-400 nm). Hyperspectral imagery is ordinarily gathered (and spoke to) as an information 3D square with spatial data gathered in the $\mathrm{X}-\mathrm{Y}$ plane, and spectral data spoke to in the Z-bearing. Hyperspectral imaging figures out how to assemble images where any single pixel is related a full spectrum in a given range. Dissimilar to multispectral methods that are now equipped for securing comparative information inside a couple of spectral groups, a constant spectrum is accessible through HSI. As exploration on explicit materials and their properties is normally distinguished by spectral marks, HSI misuses a similar goal down to the pixel level with the further preferred position of 
IJAMSR 3 (1) www.ijamsr.com CrossRef: https://doi.org/10.31426/ijamsr.2020.3.1.3013

conceivable grouping and division of the general spectral and imaging data, as for land analysis by satellite perception indicating unmistakable locales with water, mineral assets, or plant expansions[5]. No earlier information is typically required with the exception of what can be acquired solely from the dataset. The wonderful blend of data is regularly spoken to by "hypercube", a multidimensional portrayal of the acquired information along numerous tomahawks giving an image of the spatial dissemination of the watched data:
Spectroscopic (one pivot, with signal originating from reflectance, fluorescence, Raman, or some other spectroscopic test), basic (three tomahawks), and furthermore time (a potential further hub). A solitary image pixel can extend down to minuscule detail and, for HSI-based magnifying instruments, up to meters for satellite information. It ought to be noticed that the spectral segment doesn't need to be constrained to the obvious range, for example, the infrared spectral locale can be a typical decision ${ }^{[6][7] .}$
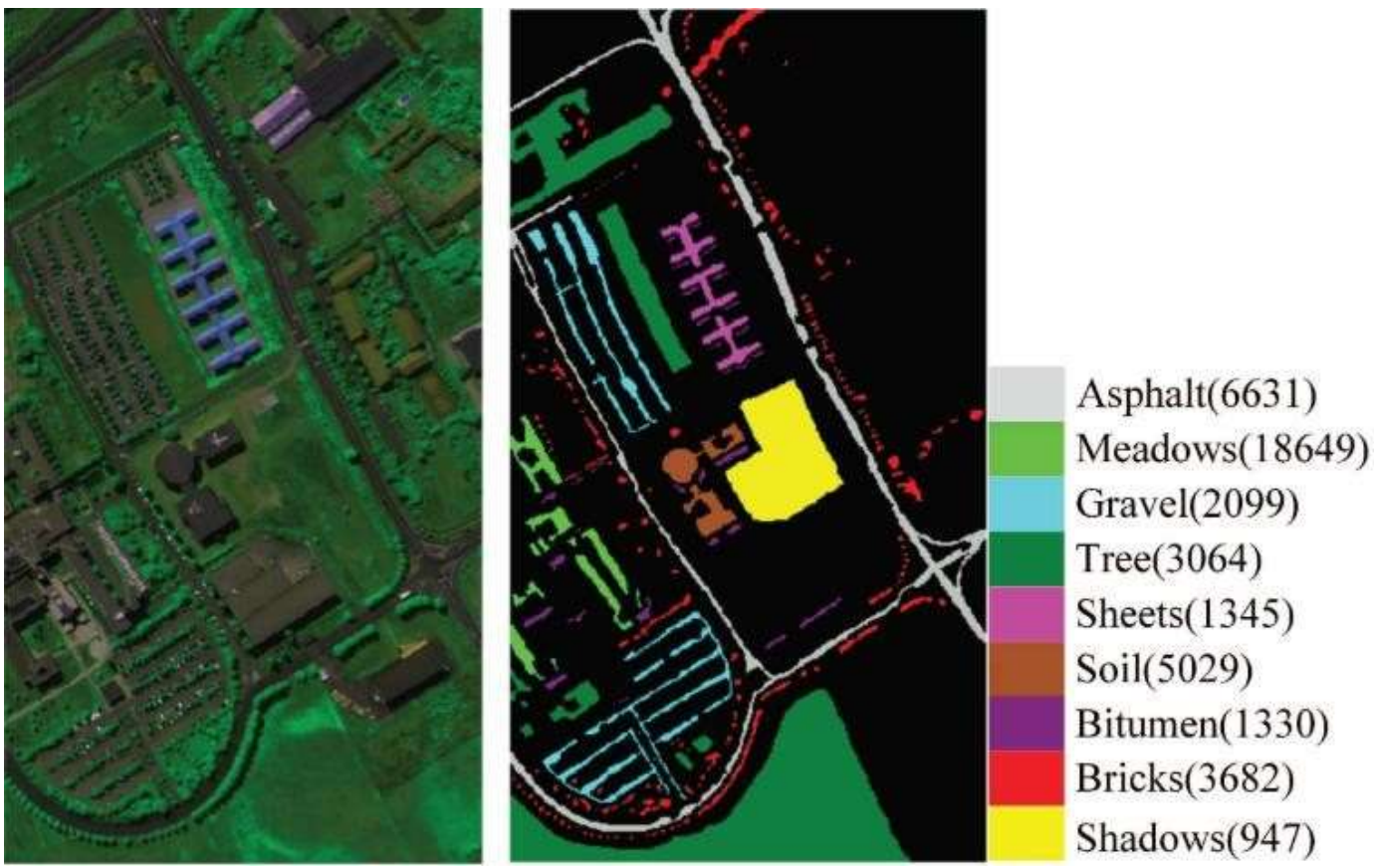

Figure 1 Hyper Spectral Imaging 
IJAMSR 3 (1) www.ijamsr.com CrossRef: https://doi.org/10.31426/ijamsr.2020.3.1.3013

\section{GAUSSIAN PROCESS}

GP strategies are famous Bayesian nonparametric methods for regression and order. An overall regression issue can be expressed as follows: $y t=f(t)+t$ where the information point $y t$ is produced by the inert function $\mathrm{f}(\mathrm{t})$ in addition to a zero- mean clamor term that we will accept to be Gaussian. The primary thought of GP regression is to utilize an endless dimensional Gaussian earlier (a GP) over the space of functions $f(t)$. This vast dimensional earlier is completely indicated by a mean function, typically thought to be indistinguishably equivalent to zero, and a covariance function $\mathrm{K}(\mathrm{t}, \mathrm{t} 0)$ that decides the earlier covariance between two diverse time focuses. The back dissemination over $\mathrm{f}(\mathrm{t})$ can be gotten by applying Bayes hypothesis. Given a lot of preparing focuses (tk, yk), it tends to be demonstrated that the back-desire $\mathrm{mf}(\mathrm{t})$ is a limited linear blend of covariance functions:

$\operatorname{Mf}(\mathrm{t})=\mathrm{X} \mathrm{kwkK}(\mathrm{t}, \mathrm{tk})$

Where the loads are linear mixes of information focuses $w \mathrm{k}=\mathrm{X} \mathrm{j}$ Akjyj
In this articulation the network An is given by the accompanying grid equation: $\mathrm{A}=(\mathrm{K}$ $+\lambda \mathrm{I})-1$

Where $\lambda$ is the fluctuation of the examining commotion and the grid $\mathrm{K}$ is acquired by assessing the covariance function for each couple of time focuses: $\mathrm{Kjk}=\mathrm{K}(\mathrm{tj}, \mathrm{tk})$ [8].

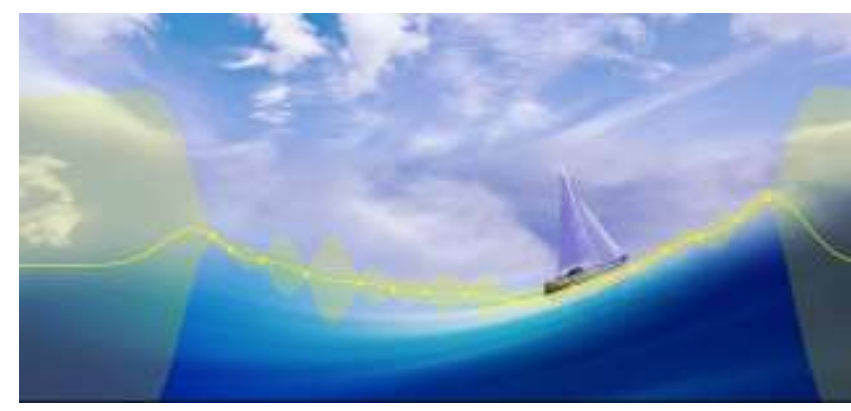

Figure 2 Gaussian Process

The objective of regression issues is to assess the function $f$, which is characterized by $y=f(x)$ and speaks to the connection between an info $\mathrm{x}$ and yield y. Evaluating this relationship is normally cultivated by obliging a class of test functions and afterward streamlining the function boundaries by utilizing preparing information to deliver the littlest mistake. One drawback of this procedure is that it just advances function boundaries and not simply the function choice, leaving the client to physically pick which functions to test. Gaussian process (GP) regressions, then again, consider every single imaginable function all the 
IJAMSR 3 (1) www.ijamsr.com CrossRef: https://doi.org/10.31426/ijamsr.2020.3.1.3013

while and figure an earlier likelihood over this set with the end goal that higher probabilities direct a more noteworthy probability that the function speaks to the information. This methodology is more adaptable, as it doesn't oblige the estimation to specific classes of functions [9][10].

\section{METHODOLOGY}

The atom swarm smoothing out (PSO) count was created while endeavoring to reproduce the lead of animals that show both individual and get-together direct, for instance, flying animals, bumble bees and fish. Each particle is seen as one expected response for the issue. The large number of particles are given a self-assertive early on region and speed and are revived by the going with conditions

$v_{i, j}^{t+1}=W v_{i, j}^{t}+c_{1} r_{1}\left(p_{i, j}-x_{i, j}^{t}\right)+c_{2} r_{2}\left(p_{g, j}-x_{i, j}^{t}\right) ;$

$x_{i, j}^{t+1}=x_{i, j}^{t}+v_{i, j}^{t+1}$

Where $\mathrm{x}$ and $\mathrm{v}$ are the position and speed of the atom I for estimation $\mathrm{j}$, independently, at time $\mathrm{t}$. $\mathrm{W}$ is the inertial weight and addresses the measure of the past speed is held while researching. Limits $\mathrm{c} 1$ and $\mathrm{c} 2$, and $\mathrm{r} 1$ and $\mathrm{r} 2$ are, independently, the weighting and sporadic factor related with the close by best particle and the global best particle

$p_{i, j}$

Nevertheless, the PSO computation is stochastic in nature and, in this manner, dependent upon the hidden conditions of the request, the improvement of the particles may vacillate and the overall ideal may not be found.

\section{PSO Property Selection}

As PSO is declared to glance through persevering spaces, it ought to be demolished for use in incorporate decision. We use a twofold type of the PSO computation, as in. The probabilities are resolved as follows.

$$
P_{i, j}=\frac{x_{i, j}^{\alpha}}{\sum_{j=1}^{n} x_{i, j}^{\alpha}},
$$

Where $\alpha$ is a scaling factor known as the decision weight. Every component is distributed with probability and the roulette wheel indicates the component to be picked. 
IJAMSR 3 (1) www.ijamsr.com CrossRef: https://doi.org/10.31426/ijamsr.2020.3.1.3013

\section{a) Hyper supernatural Data}

Reflectivity spectra were taken using an ASD spectrometer. There are 409 ghostly gatherings in the instructive assortment. The educational file is made out of 9 stone sorts routinely found in the area, as recorded in Table I.

Table 1: Rock Types Contained in the hyper supernatural Data Set.

\begin{tabular}{|l|l|}
\hline Code & Description \\
\hline \hline WRC & Water Reactive Clay \\
\hline BIF & Banded Iron Formation \\
\hline GOE & Goethite \\
\hline MAR & Martite \\
\hline SHL & West Angelas Shale \\
\hline SHN & Manganiferous Shale \\
\hline NS4 & Marker Shales \\
\hline NS3 & Volcanic Shales \\
\hline CHT & Chert \\
\hline
\end{tabular}

\section{b) Performance Metrics}

To evaluate the introduction of our strategy, we present an examination of various authentic estimations including precision, exactness, survey, F-measure, kappa and region under the ROC twist (AUC). All of the estimations were resolved as follows:

$$
\begin{gathered}
F-\text { measure }=\frac{2 \times \text { precision } \times \text { recall }}{\text { precision }+ \text { recall }} \\
\text { Kappa }=\frac{(P \times(T P+T N)-C)}{\left(P^{2}-C\right)} \\
P=(T P+T N+F P+F N) \\
C=(T P+F N) \times(T P+F P)+(F P+T N) \times(F N+T I
\end{gathered}
$$

$$
\text { Accuracy }=\frac{T P+T N}{P}
$$$$
\text { Precision }=\frac{T P}{T P+F P}
$$

$$
\text { Recall }=\frac{T P}{T P+F N}
$$

Where T P, T N, F P, F N is the amount of substantial and false positives and negatives, each remarkable estimation gets a substitute aspect of the direct of the model. Exactness is by a wide edge the most by and large used estimation in gathering considers. Regardless, as our results will show, accuracy alone likely won't be the best indication of good execution of the classifier. Thusly, in fields, for instance, information recuperation or bioinformatics, various estimations, such precision, survey 
IJAMSR 3 (1) www.ijamsr.com CrossRef: https://doi.org/10.31426/ijamsr.2020.3.1.3013

and F-measure, are usually used. Kappa is a metric that is notable in hyper unearthly (far away distinguishing) thesis and is consolidated here to allow connection. The ROC twist and the relating AUC are in like manner critical estimations used in AI. For all estimations, higher characteristics address better execution, and their most outrageous worth is 1 , by definition.
One thing to see is the manner in which the extent of precision regards in the results was commonly close diverged from F- measures. For this circumstance, exactness alone could convey ambiguous or regardless, beguiling outcomes. F-measure, joined with various estimations, allowed an all the more remarkable assessment of execution.

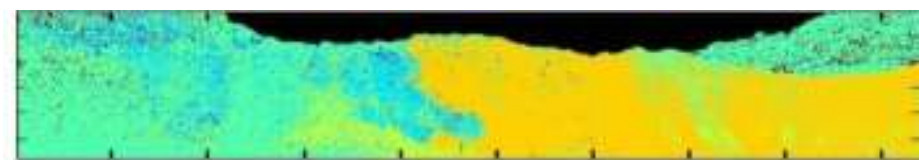

Figure 3 (A) PSO selection: 9 features

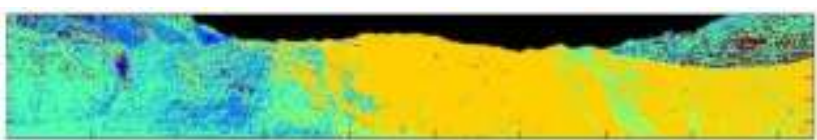

Figure 3 (B) SFS selection: 10 features

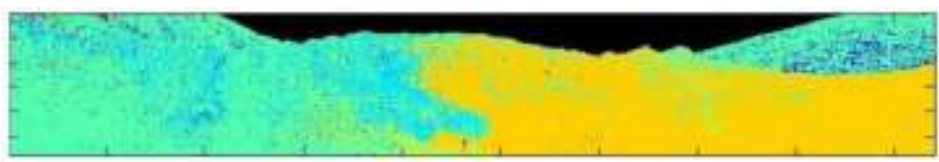

Figure 3 (C) SBS selection: 8 features

Figure 3 Examination of multi-class characterization results delivered by the various techniques. 
IJAMSR 3 (1) www.ijamsr.com CrossRef: https://doi.org/10.31426/ijamsr.2020.3.1.3013

\section{J A M S R}

\section{International Journal of}

Advanced Multidisciplinary Scientific Research (IJAMSR) ISSN:2581-4281

The results of the part assurance show that regardless, using a diminished enlightening record by picking a couple of key features we can regardless hold an agreeable level of exact request.

Whether or not the improvement in gathering execution is pretty much nothing, the diminishing in getting ready and test time for the SVM using only the picked incorporate set is astonishing. PSO gave the best performing decision of hyper ghastly features. No doubt in light of the fact that PSO has more prominent flexibility to explore the request space of likely features, stood out from SFS and SBS.

The veritable depiction results on the named set in cut off of the measure of picked social occasions. The botch is surveyed utilizing dismiss one bootstrapping with a multi-class classifier. The picked features identify with explicit gatherings and as such the physical significance of the exchange speeds in that range can be recognized. The results show that countless the noteworthy frequencies for gathering the stone sorts are arranged at the lower end of the range. Though none of the three decision methods picked a comparative component, there is evidence to suggest explicit areas where the features picked are only several gatherings isolated.

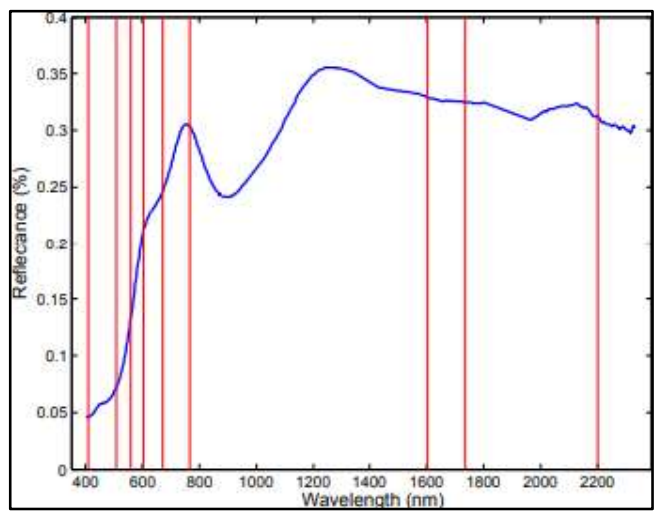

Figure 4 (a) PSO selection: 9 features

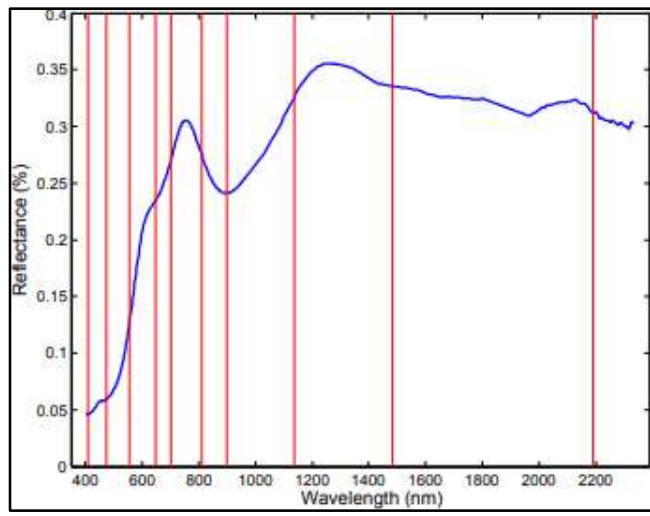

Figure 4 (b) SFS selection: 10 features

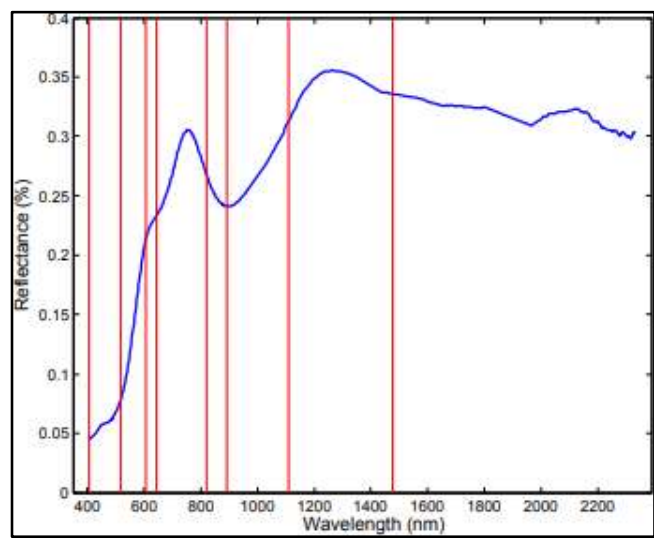

Figure 4 (c) SBS selections: 8 features

Figure 4 Comparison of supernatural features selected by the different methods for classifying martite. 
IJAMSR 3 (1) www.ijamsr.com CrossRef: https://doi.org/10.31426/ijamsr.2020.3.1.3013

\section{International Journal of}

Advanced Multidisciplinary Scientific Research (IJAMSR) ISSN:2581-4281

The dull showing up of the $\mathrm{M}$ best fragment choice is clearly appeared in the Figure. This can be clarified by the solid associations between the $\mathrm{M}$ best isolating social affairs.

They picked bundles are appeared. The gettogethers were totally perused inside a practically identical territory. Applying SFFS improved the social affair execution profoundly. They picked bundles for $\mathrm{M}=5$, which are unquestionably more spread out over the range.

The suitability of the procedures was displayed using hyper otherworldly instructive files of rock tests; a troublesome genuine issue, since the ghastly signs of metal bearing rocks are essentially equivalent to, which make course of action particularly irksome. PSO was very ground-breaking in picking features and overdue common successive decision strategies. Our preliminaries with SVM part assurance reveal that the OAD portion is significantly proper to the course of action of hyper ghastly data. It beat other for the most part used kernals, for instance, the RBF portion, and moreover kernals expressly expected to measure hyper unearthly data.

Regardless, the PSO-supported procedure isn't restricted to SVMs and can be easily contacted work in blend in with other piece techniques, for instance, Gaussian cycle backslide and course of action. Future works fuse modifying the mixing characteristics of PSO on the component assurance issue, perhaps investigating an elective decision procedure, for instance, in.

When applying band confirmation, a basic uniform taking after of the range as in starting at now gave satisfying outcomes.
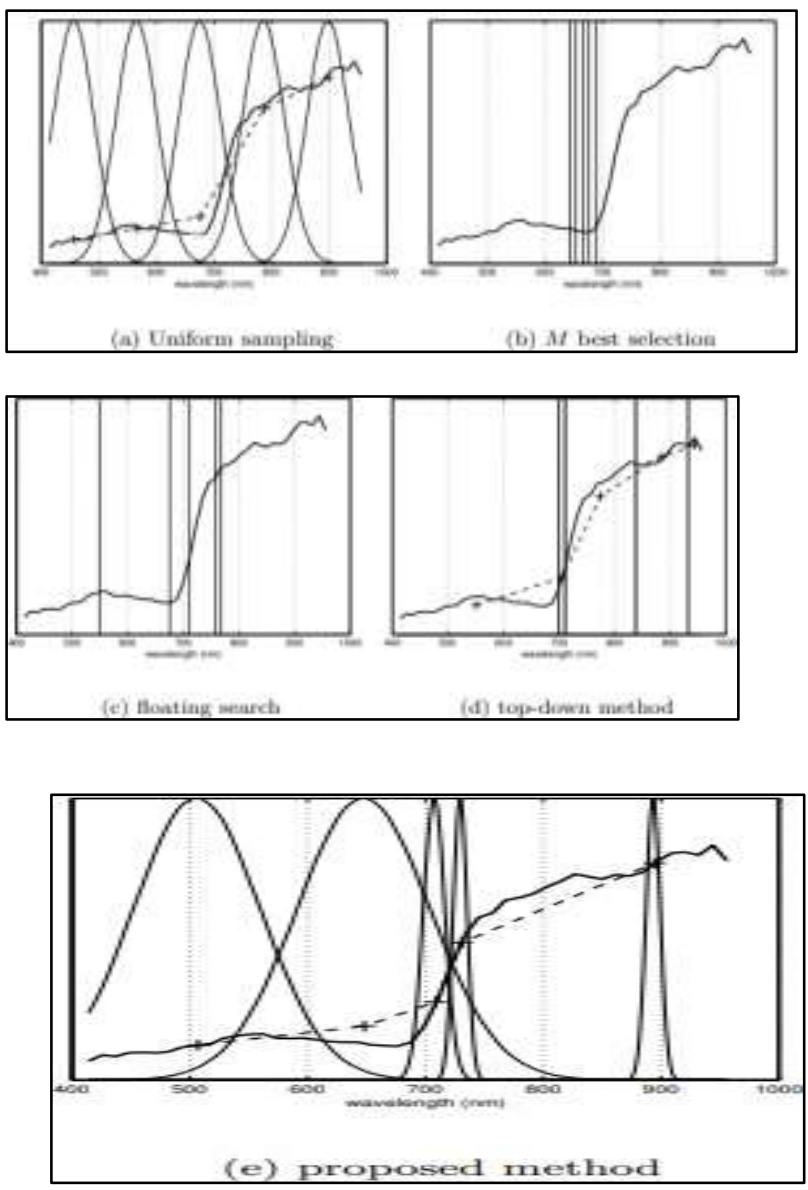

Figure 5: bands selection with different techniques. 
IJAMSR 3 (1) www.ijamsr.com CrossRef: https://doi.org/10.31426/ijamsr.2020.3.1.3013

Plan execution is only two or three percent more deplorable than with SFFS. Here, the calculation halted at estimation. The show for low estimations is adequate, anyway it separates appeared differently in relation to the SFFS for higher estimations. In the picked zones are showed up.

Results improved over the usage of SFFS, which shows that both, the spreading out over the entire range and the class reparability, are critical principles for ideal gathering. In the picked bunches are showed up for $\mathrm{M}=5$.

\section{Evaluation of Covariance Functions}

Here, we evaluate the perceptive presentation of different covariance limits. The chief analysis takes a gander at the farsighted introduction of all analyzed covariance deal with whole of all of the three authentic datasets.

The hyper limits of the GPs were smoothed out by restricting the negative log-likelihood work using semi Newton procedure. To hinder neighbourhood minima, distinctive upgrade fundamentals with unpredictable starting assumptions, inspected from $\mathrm{h}$ 10-5, $105 \mathrm{I}$, were performed. Consider the judicious introduction of different procedures on the real datasets.

Table 2: Predictive performance of different methods on all three datasets measured in $\mathrm{R}$

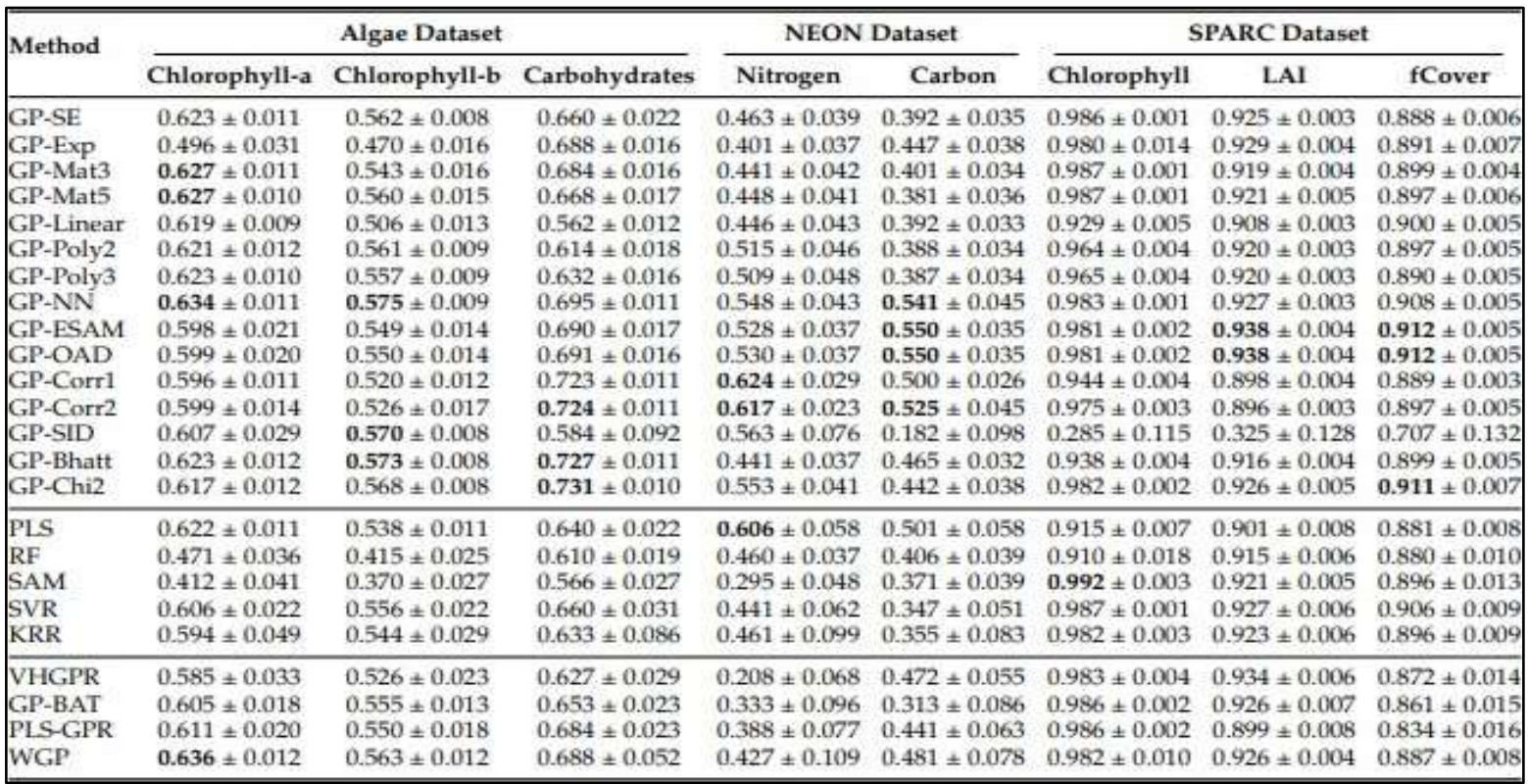


IJAMSR 3 (1) www.ijamsr.com CrossRef: https://doi.org/10.31426/ijamsr.2020.3.1.3013

For assist vector with backsliding and kernal edge backslide, we utilized the less mind boggling apparatus stash utilization with squared exponential kernals, which have been used by past assessments for aggregation limit assessment. We moreover contrast the outcomes and top tier approaches for aggregation limit desire, i.e., GP-BAT, VHGPR, PLS-GPR, and WGP. VHGPR was completed using the less intricate device stash; GP-BAT and WGP was executed using GPML device compartment; and PLS-GPR was executed using the simpler and the sim Feat toolboxs.

Correlation among unearthly and other covariance work: The outcomes show that ghostly covariance limits played out the best.
The non-fixed covariance works with everything taken into account beat the fixed covariance limits (checking the squared exponential limit which was used by an enormous segment of the past assessments). This is a result of the way that the Euclidean division between the spectra isn't satisfactory estimation for similarity for unearthly information. Our outcomes exhikernal that while applying Gaussian processes for aggregation limit assessment, instead of basically utilizing the default squared exponential covariance fill in as done by past assessments; it is savvy to use simulation assurance techniques, for instance, cross endorsement, to pick the best non-fixed covariance work.

Table 3: Performance of Prediction

\begin{tabular}{|c|c|c|c|c|c|c|c|c|}
\hline \multirow{2}{*}{ Method } & \multicolumn{3}{|c|}{ A Isae Dataset } & \multicolumn{2}{|c|}{ NEON Dataset } & \multicolumn{3}{|c|}{ SPARC Dataset } \\
\hline & Chlerophyll-a & Chlarophyll-b & Carbohydrates & Nitrogen & Carbon & Chlorophyll & 1.A1 & fCover \\
\hline CP-SE & $9.716 \pm 0.138$ & $0.323+0.003$ & $8.701+0.278$ & $0.275+0.012$ & $1.712+0.057$ & $2.134+0.106$ & $0.457 \neq 0.008$ & $0.115 \pm 0.003$ \\
\hline & 11.2944 & $5+0,0015$ & $43+0$ & $0+0$ & $32+$ & 60. & 0.447 & \\
\hline data & $0.667+0.135$ & $0.330+0.006$ & $8.397+0.202$ & $2861+0.013$ & $1.700+0.0554$ & 0.070 & 0.475 & 0.100 \\
\hline GPMats & $9.662+0.136$ & $0.323 \neq 0.005$ & $8.604 \pm 0.216$ & $279+0.013$ & $1.730=0.054$ & $2.059+0.075$ & $0.467 \neq 0.014$ & 0,110 \\
\hline Glo-1 inear & $9.766+0.123$ & $0.343+0.005$ & $9.896+0.179$ & & 31. & & 0.504 & 03 \\
\hline & $9.736+0.152$ & $3+0.8$ & $0.2 \mathrm{N6}+1$ & 4 & 3 & & 63. & \\
\hline Gar & $0,717+0.1$ & $0.325+0.6$ & $9.060+0.2$ & $3+$ & $8+$ & 3 & 6 & 22 \\
\hline $\mathrm{GF}$ & $9.575+0.139$ & $0.318+0.003$ & $8.238+0.144$ & $0.251+0.014$ & $1.517+0.094$ & $2326+0.069$ & $0.450+0.009$ & $0.104=$ \\
\hline CFFAM & $\begin{array}{l}100.035 \\
10017\end{array}$ & $\begin{array}{l}0.327 \\
0.327+0.005\end{array}$ & $\begin{array}{l}8.311+0.223 \\
8302+0.220\end{array}$ & $\begin{array}{l}0.256=0.011 \\
0.255\end{array}$ & $\begin{array}{l}1.478=0.6 \\
1.478+04\end{array}$ & $\begin{array}{l}2.509 \\
2.505\end{array}$ & $\begin{array}{l}0.416 \\
0.416\end{array}$ & $\begin{array}{l}0.101=0.003 \\
0.102=0003\end{array}$ \\
\hline cise & $10.0062=$ & o.a3s + o. & $7.850+0.1$ & $0.220+0.000$ & 1.554 & 4.221 & 10 & $0.114+0.002$ \\
\hline$G$ & 10.0 & 6 & 9 & 18 & 1.543. & 28 & $0.537+0$. & 0.110 \\
\hline $\mathrm{ki}$ & 9.93 & & 3 & 3 & $2.0:$ & 158 & 1.362 & 0.181 \\
\hline$\Leftrightarrow$ & 9.71 & 10 & o & $0.278+0$ & $1.600=0$ & $\therefore 0$ & $0.483+0$ & $0.100+0003$ \\
\hline CaPchi2 & $9.792+0.158$ & $0.320+0.003$ & $7.745+0.147$ & $0.253+0.014$ & $1.690+0.088$ & $2.3999+0.135$ & $0.454+0.015$ & $0.102+0.004$ \\
\hline MLS & $9.773+0.36$ & $375+0,005$ & $101+0.76$ & $247+0.026$ & $1,6683+6$ & $5.213 \neq 0.224$ & 0.525 & 0.120 \\
\hline & & & & & & & & \\
\hline & 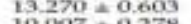 & & & & & & & \\
\hline 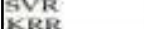 & & & & & & & & \\
\hline KRR & $10.119-0.607$ & $0.230+0.011$ & $9.236+1.203$ & $0.287+0.040$ & 1. $906+0.201$ & $2.370+0.210$ & $0.46-4=0.020$ & $0.116+0.005$ \\
\hline & 10.241 & & & 8 & & & & 17 \\
\hline & 9 & & & & 2 & & & \\
\hline$P I$ & $9.878+0$ & +1 & E & $12+$ & $1.72 n+$ & 21 & 0.47 & 0.12 \\
\hline & $9.659 \neq 0.132$ & $0,326 \neq 0,004$ & $8,380+0,700$ & $0.207+0.054$ & $1.736 \neq 0.259$ & $2.36+8+0,5005$ & $0.45 .3+0.013$ & $0.115+0.004$ \\
\hline
\end{tabular}


IJAMSR 3 (1) www.ijamsr.com CrossRef: https://doi.org/10.31426/ijamsr.2020.3.1.3013

The correlation of GP supported procedures with SAM for SPARC dataset's Chlorophyll Figure will be broke down again in the third assessment. Despite the top tier procedures recorded in the table, we similarly explored different avenues regarding a progressing significant learning supported biophysical/biochemical limit conjecture arrange, called Deep Spectra.
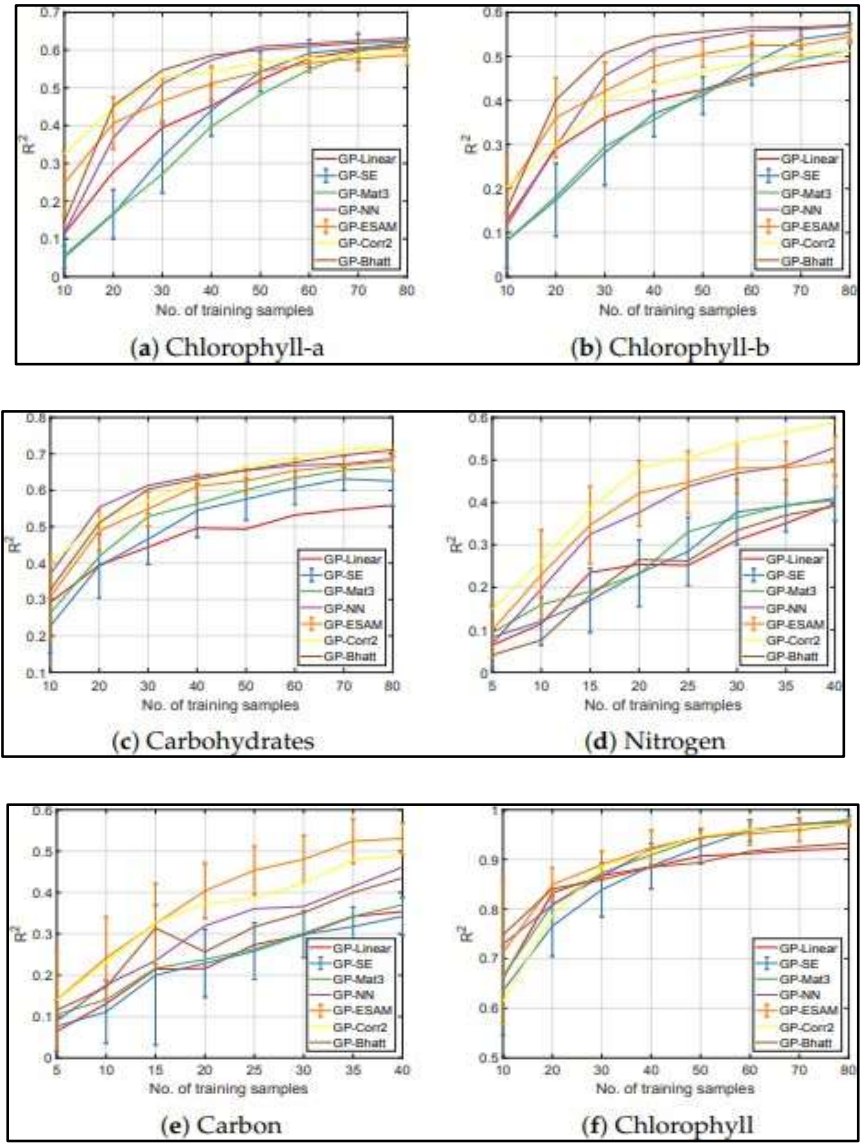

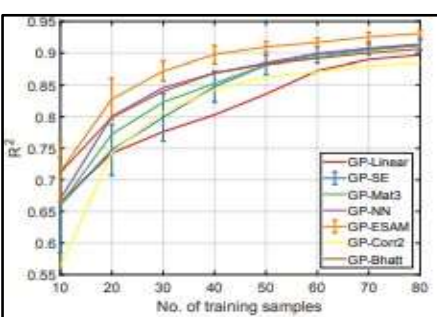

(g) LAI

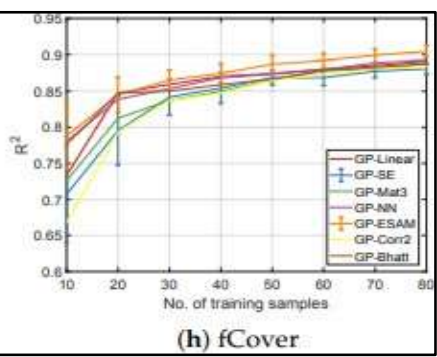

(h) fCover

Figure 6: Performance as a function of training set size. 
IJAMSR 3 (1) www.ijamsr.com CrossRef: https://doi.org/10.31426/ijamsr.2020.3.1.3013

The explicit system con Figure tried was the one used to envision corn protein in the Deep Spectra thesis. In our tests, we found that the approval adversity didn't consolidate, regardless, when the preparation setback joined, for NEON dataset (the most diminutive dataset), and for Chlorophyll-a and Chlorophyll-b in Algae dataset. For rest of the limits, the simulation made vulnerable outcomes. The $\mathrm{R} 2$ of gauge for Carbohydrate in Algae dataset was $0.6459 \pm$ 0.0392 and the R 2 of desire for Chlorophyll, LAI, and $\mathrm{f}$ Cover in SPARC dataset were $0.9114 \pm 0.0179,0.8848 \pm 0.0149$, and $0.8783 \pm 0.0170$, independently. This clearly shows Deep Spectra simulation was overfitting on our datasets. In the accompanying examination, we test how the introduction of the models shifts with getting ready set size. The results are showed up in Figure. Since, both R2 and RMSE execution estimations demonstrated reliable outcomes in the primary trial, just R2metric is represented in the remainder of the thesis. The presentation twists were obtained by using a balanced sort of reiterated 10 cover cross-endorsement. By changing the size of the subset, the presentation as breaking point of arranging set size was gotten. This cycle ensures that the size of the test set is same, in spite of the way that the size of the preparation set is changing, with the objective that the outcomes got from models arranged on preparing sets of different sizes can be pondered. In this investigation, we didn't extensively cover the aggregate of the covariance limits, yet just picked an operator set. Just standard deviations of the squared exponential covariance work and the exponential supernatural edge arranged are showed up for then again relationship. The plots again show that the non-fixed limits are better. Generally speaking, we find that the gap in execution between fixed covariance limits and non-fixed covariance limits is greater when the preparation set size is close to nothing.

This gap kernal by kernal restricts as the amount of preparing models is extended. This shows non-fixed covariance limits (ghastly limits explicitly) are best for biophysical conjecture when the ground truth is obliged. 
IJAMSR 3 (1) www.ijamsr.com CrossRef: https://doi.org/10.31426/ijamsr.2020.3.1.3013

The outcomes of the third investigation that utilizes the built dataset to show the effects of light assortments and size of preparing set. The mean R 2 estimation in excess of 30 repeats of 10-wrinkle cross endorsement is showed up in the Figure. We have used 9 built datasets gained by setting $\theta$ var regards from $0^{\circ}$ to $90^{\circ}$. Each of those used a comparative technique as the last investigation to be obtained execution under fluctuating preparing set size. The outcomes exhikernal that non-fixed covariance limits (explicitly ghostly covariance limits) perform better under compelled ground truth just as when illumination assortment is high in the preparation set. One dumbfounding outcome got in that SAM performed better than Gaussian cycle supported system while envisioning chlorophyll in SPARC dataset.

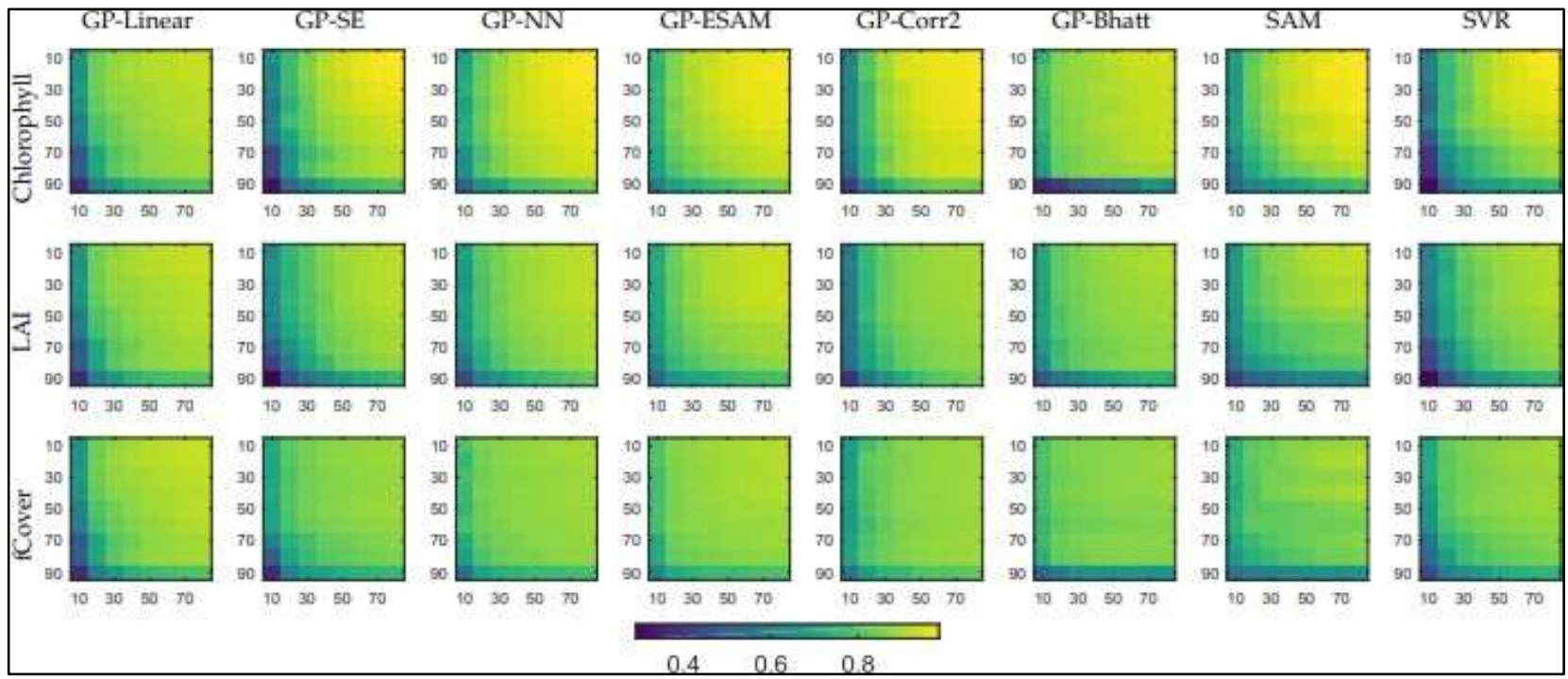

Figure 7: Mean prescient R2 as capacity of preparing set size and light variations evaluated on mimicked dataset. 
IJAMSR 3 (1) www.ijamsr.com CrossRef: https://doi.org/10.31426/ijamsr.2020.3.1.3013

Shows that when the preparation set is confined or lighting up assortment is high the non-fixed covariance limits outperform the SAM. This is in light of the fact that SAM, which is basically a nearest neighbor method with cosine resemblance as partition metric, needs tremendous game plan of preparing information to work fittingly as it makes hard decision to designate the test to the closest preparing set example. If the gap between the preparation set is tremendous, the bumble is high for SAM yet GP can get comfortable with a smooth limit between the gaps to diminish the Figure botch.

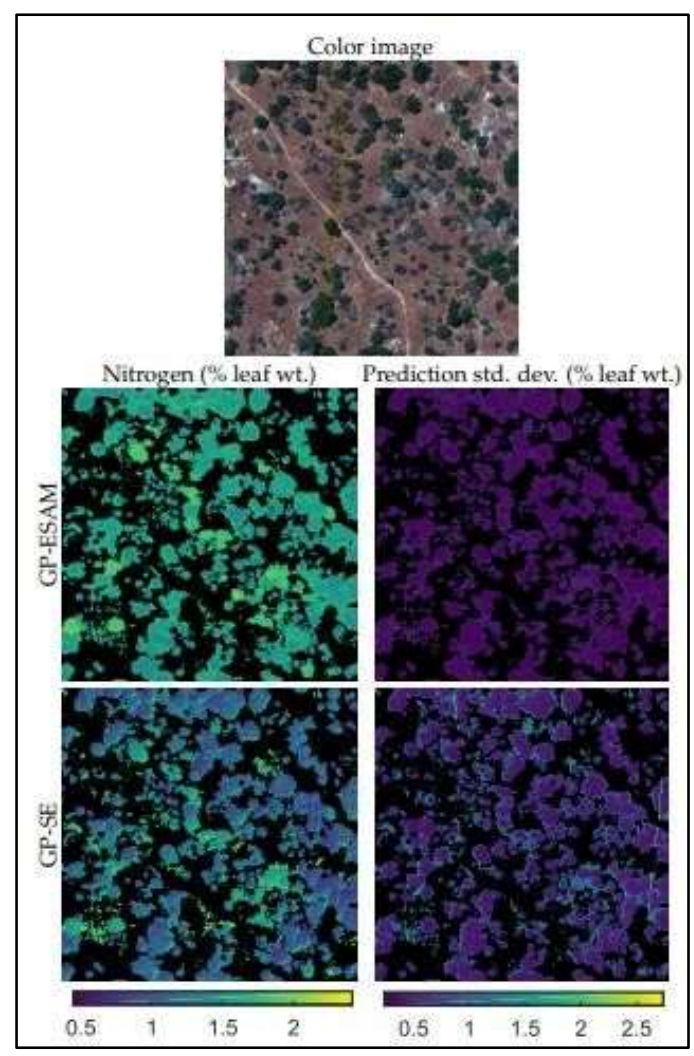


IJAMSR 3 (1) www.ijamsr.com CrossRef: https://doi.org/10.31426/ijamsr.2020.3.1.3013

Figure 8: Gaussian cycles (GP) exponential extraordinary point planned 200 what's more, 50 preparing tests were created by discretionarily picking tests from the NEON dataset and going it through the 4SAIL model. The limits of 4SAIL simulation were set as follows. The daylight supported pinnacle, the sun arranged azimuth and the extent of characteristics for the overview apex and the audit azimuth from which they were indiscriminately inspected from were obtained from the meta- information in the test picture record. Various limits were reliably tested erratically from a range-leaf district record: 0.1 to 4.0, typical leaf point: $10^{\circ}$ to $80^{\circ}$, issue region limit: 0.01 to 1.0 , and soil splendor factor: 0.1 to 1.0 .

\section{Evaluation of Multitask Gaussian Processes}

We try different things with two aggregation limits from each dataset immediately. We acknowledge that we have confined ground truth for the fundamental aggregation limit yet have greater measure of ground truth for the discretionary aggregation limit. We follow a comparative appraisal framework as in the past trials to get execution as a component of fundamental aggregation limit's preparation set size.

The primary difference is that in each crossendorsement inside $30 \quad$ self-assertive fundamentals simply the ground truth of the basic aggregation limit is remained alive from the preparation overlap while the sum of the arrangement overlay ground truth of the discretionary aggregation limit is kept. So each simulation is set up on spectra in the preparation overlap which have helper aggregation limit ground truth for the sum of its examples anyway fundamental biophysical limit ground truth for only a subset has. To reliably think play out various assignments models; we set the covariance ability to ESAM all

through the tests. To guarantee that and also $\mathbf{K}_{\mathrm{M} \times \mathrm{M}}$ for MTGP2, are PSD, they are parameterized as

$\sum_{i=1}^{r} \mathbf{a}_{i} \mathbf{a}_{i}^{\mathrm{T}}+c^{2} \mathbf{I}_{\mathrm{M} \times \mathrm{M} .} \quad \begin{gathered}\mathbf{a}_{i} \forall i \\ \text { where }\end{gathered}$ dimensional vectors, $\mathrm{c}$ is a scalar, $\mathrm{r}$ is a number whose value can run from 1 to $\mathrm{M}$. This assessment generally conveys a PSD grid considering the way that the corner to corner of the conjecture generally has higher size than the rest of the parts. 
IJAMSR 3 (1) www.ijamsr.com CrossRef: https://doi.org/10.31426/ijamsr.2020.3.1.3013

$\mathbf{a}_{i}{ }^{\prime} \mathrm{s}$ What's more, $\mathrm{c}$ is found out from the information with other hyper boundaries. The assessment of $r$ controls the position and the amount of hyper limits related with and $\underset{\text { During }}{\mathbf{K}_{\text {planning, we }}^{f}} \stackrel{\Sigma_{\mathrm{M} \times \mathrm{M}_{\mathrm{M}}}}{\boldsymbol{\Sigma}_{\mathrm{M}}}$ separate models by exclusively setting the assessment of $r$ to values in the range 1 to $\mathrm{M}$ and tuning the hyper limits of the simulation using a comparable framework as the one used for GPs in past tests.

For Algae dataset, perform different undertakings learning of chlorophyll-an and chlorophyll-b was valuable yet adjusting them two with starch was certainly not. The joint displaying of leaf nitrogen and leaf carbon apparently was useful in NEON dataset. For SPARC dataset, perform different errands learning of leaf locale record and incomplete aggregation covers performed even better either didn't benefit by joint demonstrating with leaf chlorophyll content.

This is typical since leaf zone record and incomplete aggregation spread are known to be associated at this point there is no close relationship of either with the leaf chlorophyll substance. Right when the fundamental aggregation limit's preparation set size is sufficiently gigantic, we see that there is no expansion from using plays out various assignments GP. The best performing simulation and any simulation which was unquestionable not measurably exceptional comparable to the best simulation (two example t-test, $\alpha=0.01$ ) have been highlighted in the table. 
IJAMSR 3 (1) www.ijamsr.com CrossRef: https://doi.org/10.31426/ijamsr.2020.3.1.3013

I J A M S R

\section{International Journal of}

Advanced Multidisciplinary Scientific Research (IJAMSR) ISSN:2581-4281

Table 4: Comparison of GP and multitask GP for aggregation parameter estimation

Performance measured by R2

\begin{tabular}{|c|c|c|c|c|}
\hline \multirow[b]{2}{*}{ No. Samples } & \multicolumn{4}{|c|}{ Algae Dataset } \\
\hline & 10 & 30 & 50 & 70 \\
\hline \multirow{4}{*}{$\begin{array}{l}\text { GP } \\
\text { MTGP1 } \\
\text { MTGP2 }\end{array}$} & \multicolumn{4}{|c|}{ Primary: Chlorophyll-a, Secondary: Chlorophyll-b } \\
\hline & $0.246 \pm 0.082$ & $0.475 \pm 0.055$ & $0.538 \pm 0.044$ & $\mathbf{0 . 5 8 3} \pm 0.028$ \\
\hline & $0.438 \pm 0.031$ & $0.546 \pm 0.024$ & $0.555 \pm 0.031$ & $0.574 \pm 0.039$ \\
\hline & $0.412 \pm 0.039$ & $0.518 \pm 0.043$ & $0.564 \pm 0.024$ & $0.583 \pm 0.024$ \\
\hline \multirow{5}{*}{$\begin{array}{l}\text { GP } \\
\text { MTGP1 } \\
\text { MTGP2 }\end{array}$} & \multicolumn{4}{|c|}{ Primary: Chlorophyll-b, Secondary: Chlorophyll-a } \\
\hline & $0.192 \pm 0.071$ & $0.435 \pm 0.071$ & $0.492 \pm 0.043$ & $0.528 \pm 0.024$ \\
\hline & $0.444 \pm 0.039$ & $0.493 \pm 0.039$ & $0.519 \pm 0.028$ & $0.528 \pm 0.025$ \\
\hline & $0.428 \pm 0.039$ & $0.499 \pm 0.034$ & $0.530 \pm 0.028$ & $0.539 \pm 0.020$ \\
\hline & \multicolumn{4}{|c|}{ NEON Dataset } \\
\hline \multirow[t]{2}{*}{ No. Samples } & 5 & 15 & 25 & 35 \\
\hline & \multicolumn{4}{|c|}{ Primary: Nitrogen, Secondary: Carbon } \\
\hline GP & $0.115 \pm 0.074$ & $0.330 \pm 0.103$ & $0.475 \pm 0.072$ & $0.505 \pm 0.055$ \\
\hline MTGP1 & $0.094 \pm 0.086$ & $0.462 \pm 0.077$ & $0.493 \pm 0.051$ & $0.514 \pm 0.050$ \\
\hline \multirow[t]{2}{*}{ MTGP2 } & $0.029 \pm 0.033$ & $0.412 \pm 0.087$ & $0.499 \pm 0.068$ & $0.517 \pm 0.047$ \\
\hline & \multicolumn{4}{|c|}{ Primary: Carbon, Secondary: Nitrogen } \\
\hline GP & $0.139 \pm 0.102$ & $0.341 \pm 0.109$ & $0.469 \pm 0.057$ & $0.513 \pm 0.053$ \\
\hline MTGP1 & $0.364 \pm 0.116$ & $0.465 \pm 0.052$ & $0.503 \pm 0.055$ & $0.530 \pm 0.044$ \\
\hline \multirow[t]{2}{*}{ MTGP2 } & $0.326 \pm 0.129$ & $0.495 \pm 0.060$ & $0.518 \pm 0.050$ & $\mathbf{0 . 5 2 2} \pm 0.040$ \\
\hline & \multicolumn{4}{|c|}{ SPARC Dataset } \\
\hline \multirow[t]{2}{*}{ No. Samples } & 5 & 10 & 15 & 20 \\
\hline & \multicolumn{4}{|c|}{ Primary: LAI, Secondary: fCover } \\
\hline GP & $0.615 \pm 0.099$ & $0.784 \pm 0.045$ & $0.851 \pm 0.023$ & $0.870 \pm 0.023$ \\
\hline MTGP1 & $0.768 \pm 0.048$ & $0.806 \pm 0.033$ & $0.814 \pm 0.076$ & $0.836 \pm 0.020$ \\
\hline \multirow[t]{2}{*}{ MTGP2 } & $0.771 \pm 0.047$ & $0.811 \pm 0.016$ & $0.827 \pm 0.014$ & $0.847 \pm 0.014$ \\
\hline & \multicolumn{4}{|c|}{ Primary: fCover, Secondary: LAI } \\
\hline GP & $0.569 \pm 0.110$ & $0.762 \pm 0.062$ & $0.822 \pm 0.047$ & $0.852 \pm 0.015$ \\
\hline MTGP1 & $0.738 \pm 0.058$ & $0.809 \pm 0.022$ & $0.825 \pm 0.018$ & $0.845 \pm 0.014$ \\
\hline MTGP2 & $0.745 \pm 0.050$ & $0.799 \pm 0.025$ & $0.828 \pm 0.018$ & $0.838 \pm 0.017$ \\
\hline
\end{tabular}


IJAMSR 3 (1) www.ijamsr.com CrossRef: https://doi.org/10.31426/ijamsr.2020.3.1.3013

Table 5: Examination of GP and perform various tasks GP for conglomeration boundary forecast (negative outcomes). Execution estimated by R2.

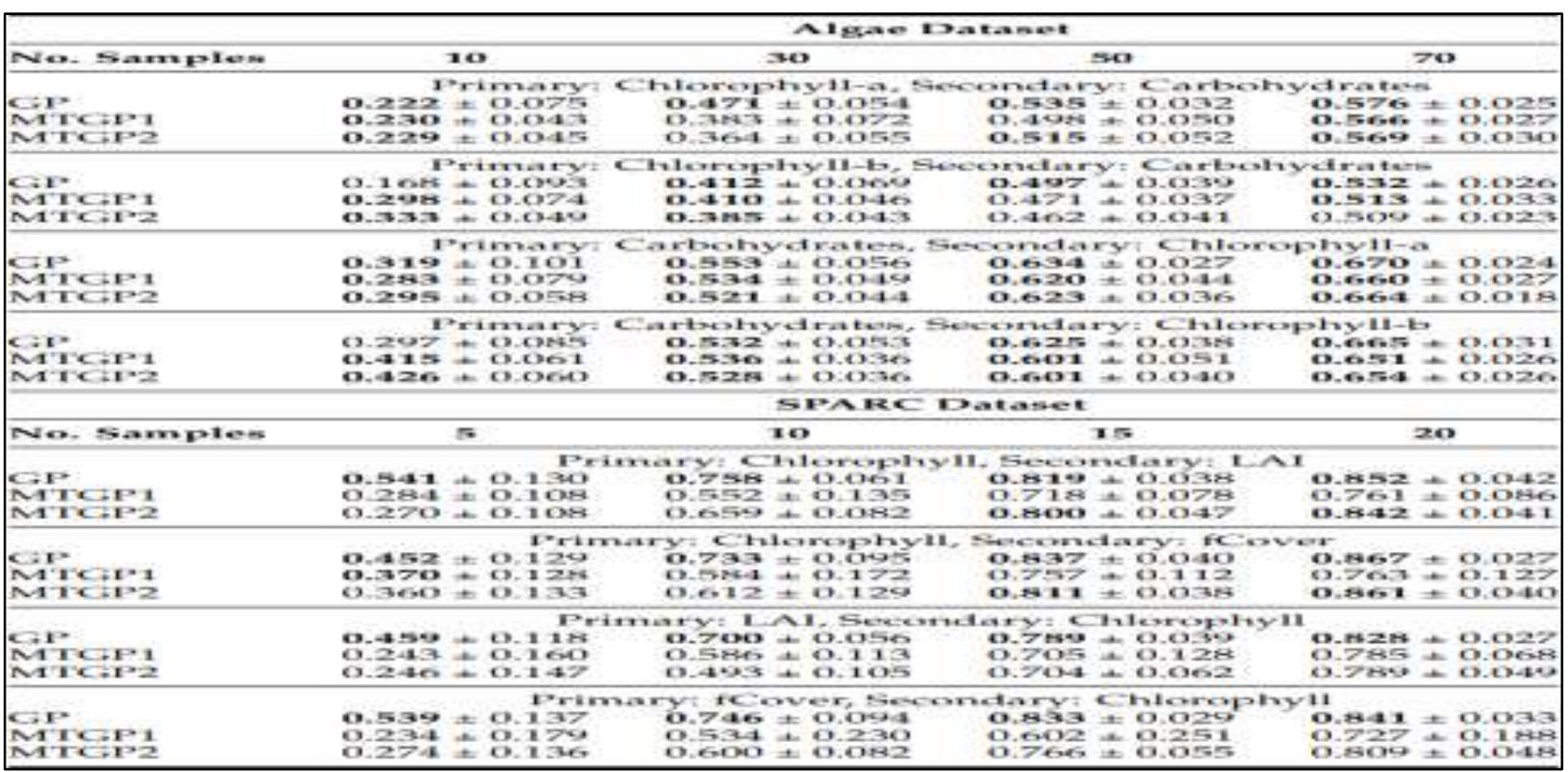

Presently, we explore whether perform various tasks learning can be utilized to improve the expectation of simulation when the enlightenment variety in the training dataset is high. For this, we again use the manufactured dataset. We see that perform multiple tasks GP beats single-task GP, at the point when either preparing set size is little and additionally light varieties is high.

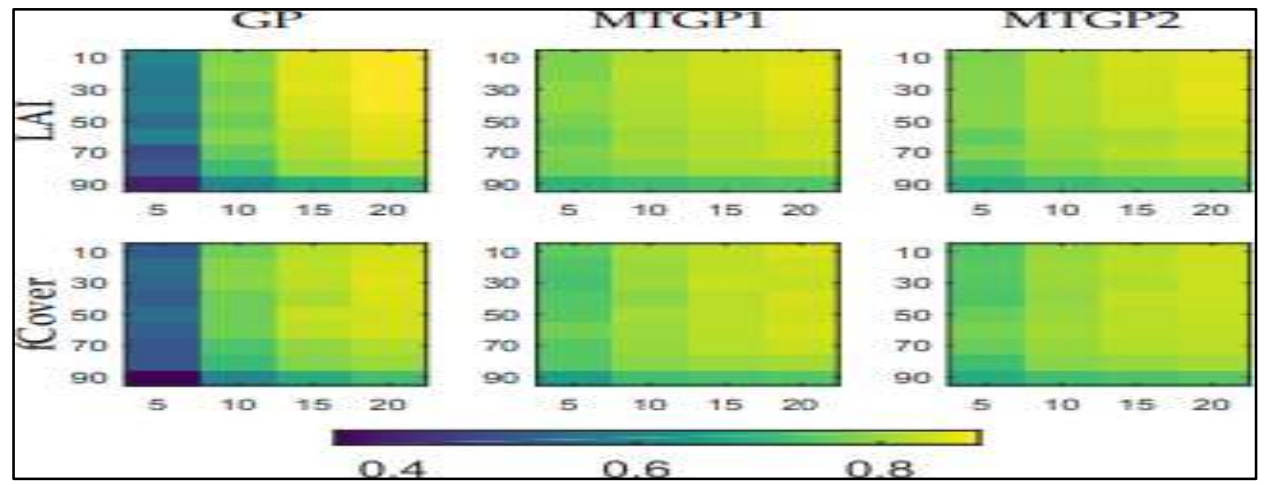

Figure 9: Mean prescient R2 of perform various tasks GP as capacity of preparing set size (xhub) and brightening varieties (y-hub) assessed on mimicked dataset. 
IJAMSR 3 (1) www.ijamsr.com CrossRef: https://doi.org/10.31426/ijamsr.2020.3.1.3013

Since attempts various things with sets of aggregation limits in Algae and SPARC dataset didn't show that every one of the three aggregation limits in the dataset are associated, we didn't attempt various things with exhikernaling three aggregations restricts along with play out different undertakings learning. Regardless, a comparative system can be used to learn more than two aggregation limits together.

\section{CONCLUSION}

This paper reviews several procedures of hyperspectral image classification using Gaussian process, including kernel level analysis of hyper spectral image. With the growth of hyperspectral image expertise, hyperspectral image grouping has been broadly used. Prevailing concepts and methods still have certain restrictions for more problematical hyperspectral image classification. Therefore, investigating further directed hyperspectral image classification methods will be a significant investigation course in the forthcoming. Also this paper investigated past works identified with analyses and sensing of hyperspectral images are significant portion of hyperspectral image processing using Gaussian process. The controlled and unsubstantiated grouping methods described in this paper have their corresponding benefits to varying gradations. Supervised classification entails a convinced quantity of certain parameters will affect the grouping outcomes have an impact. Therefore, based on dissimilar application necessities, combined with the acquirement of hyperspectral images with enormous statistics, numerous approaches need to be joined with each other in order to accomplish the anticipated grouping outcome. Also successfully implemented the design of particle swarm optimization based gaussian process and analyzing hyper spectral reflectance.

\section{References}

1. Felipe Tobar Universidad de Chile "Bayesian Nonparametric Spectral Estimation".arXiv:1809.02196v2 [stat.ML] 12 Jan 2019.

2. Gregory R. Romanchek ,Zheng Liu,Shiva Abbaszadeh. "Kernel-based Gaussian process for anomaly detection in sparse gamma-ray data". January 23,2020https://doi.org/10.1371/journal.pon e. 0228048 . 
IJAMSR 3 (1) www.ijamsr.com CrossRef: https://doi.org/10.31426/ijamsr.2020.3.1.3013

3. Luca Ambrogioni Eric Maris. "Integral Transforms from Finite Data: An Application of Gaussian Process Regression to Fourier Analysis". Proceedings of the 21st International Conference on Artificial Intelligence and Statistics (AISTATS) 2018, Lanzarote, Spain. PMLR: Volume 84. Copyright 2018 by the author(s).

4. Haitao Liu, Yew-Soon Ong, Fellow, IEEE, Xiaobo Shen, and Jianfei Cai, Senior Member, IEEE. "When Gaussian Process Meets Big Data: A Review of Scalable GPs" 2019. arXiv:1807.01065v2.

5. Kai ChenEmail authorTwan van LaarhovenJinsong ChenElena Marchiori. "Incorporating Dependencies in Spectral Kernels for Gaussian Processes". Joint European Conference on Machine Learning and Knowledge Discovery in Databases ECML PKDD 2019: Machine Learning and Knowledge Discovery in Databases pp 565$581 \mid$

6. Stefano Selci. Institute for Photonics and Nanotechnologies, ARTOV C.N.R., Via del Fosso del Cavaliere 100, 00133 Roma,

7. Italy; Stefano.Selci@cnr.it"The Future of Hyperspectral Imaging". J. Imaging 2019, 5, 84; $\quad$ doi:10.3390/jimaging5110084 www.mdpi.com/journal/jimaging.

8. Dongyi Wang, Robert Vinson, Maxwell Holmes, Gary Seibel, Avital Bechar, Shimon Nof \& Yang Tao. "Early Detection of Tomato Spotted Wilt Virus by Hyperspectral Imaging and Outlier
Removal Auxiliary Classifier Generative Adversarial Nets (OR- AC-GAN)" 2019. Open access.

9. Peng Fu1, Katherine Meacham-Hensold1, Kaiyu Guan and Carl J. Bernacchi1. "Hyperspectral Leaf Reflectance as Proxy for Photosynthetic Capacities: An Ensemble Approach Based on Multiple Machine Learning Algorithms".

https://doi.org/10.3389/fpls.2019.00730.

10. Utsav B. Gewali.2019. "Gaussian Processes for Vegetation Parameter Estimation from Hyperspectral Data with Limited Ground Truth". https://doi.org/10.3390/rs11131614.

11. Maxim Ziatdinov, Dohyung Kim, Sabine Neumayer, Rama K. Vasudevan, Liam Collins, Stephen Jesse, Mahshid Ahmadi \& Sergei V. Kalinin. "Imaging mechanism for hyperspectral scanning probe microscopy via Gaussian process modelling. Open access. 2020.

12. Wenjing $\mathrm{L}$ and Xiaofei Wang. "Overview of Hyperspectral Image Classification". Volume 2020 Article ID 4817234 | 13 pages | https://doi.org/10.1155/2020/4817234.

13. Liu Z, Abbaszadeh S, Sullivan CJ. Spatialtemporal modeling of background radiation using mobile sensor networks. PloS one. 2018 Oct 19;13(10):e0205092. pmid:30339704.

14. J. Hensman, N. Durrande, and A. Solin. Variational fourier features for Gaussian processes. Journal of Machine Learning Research, 18(151):1-52, 2018.

15. R. Boloix-Tortosa, J. J. Murillo-Fuentes, F. J. Payán- Somet, and F. Pérez-Cruz. Complex Gaussian processes for regression. IEEE Transactions on Neural Networks and Learning Systems, 29(11):5499- 5511, 2018. 